\title{
Shear Lag Solution for Structurally Attached Active Sensors
}

\author{
Giola Santoni-Bottai $^{1 \mathrm{a}}$, Victor Giurgiutiu ${ }^{\mathrm{a}}$ \\ ${ }^{a}$ Department of Mechanical Engineering, University of South Carolina, 300 Main St. Columbia, SC \\ 29208 USA
}

\begin{abstract}
Piezoelectric wafer active sensors (PWAS) have been used as actuators in structural health monitoring system of beams, plates, and truss elements. This paper presents a shear lag solution for the transfer of stress and strain between a structurally attached piezoelectric wafer active sensor and the support structure. We will derive a shear lag solution not limited to the low frequency approximation, i.e., a generic solution. Both the low frequency approximation and the generic solution will be applied to the computation of PWAS-structure tuning curves.
\end{abstract}

Keywords: structural health monitoring, Lamb wave, shear lag, piezoelectric sensors, tuning curves.

\section{Introduction}

Under electric excitation, the PWAS undergoes oscillatory contractions and expansions which are transferred to the structure through the bonding layer and thus excite Lamb waves into the structure. The transducer is bonded to the plate with an adhesive layer. The contractions and expansions of the PWAS are transmitted to the material through the bond layer. Due to the reciprocity of the piezoelectric properties, the PWAS can be also used to detect Lamb waves and transform them to electric signals. Crawley and de Luis [1] developed an analytical model of the mechanical coupling of segmented piezoelectric actuators to the dynamics of a structural member. The configuration they studied was of two piezoelectric elements bonded by a finite bonding layer to an elastic structure. The solution proposed was the classic shear lag solution. They showed that when the shear lag goes to zero, the solution reduces to the simpler model of the perfectly bonded piezoelectric wafer. They assumed that the strain distribution in the piezoelectric wafer was a linear distribution across the thickness, further more they assumed that the strain distribution across the structure could be a linear Bernoulli-Euler type or a uniform extensional strain. The shear lag parameter $\Gamma$ was found to depend on the modal repartition number $\alpha$ which took the value $\alpha=1$ for symmetric (i.e., axial) excitation and $\alpha=3$ for antisymmetric (i.e., flexural) excitation. This initial analysis was further detailed by Crawley and Anderson [2]. Giurgiutiu [3] extended Crawley and de Luis [1] and Crawley and Anderson [2] theory to the case of only one piezoelectric element bonded to the thin-wall structure by calculating the total effect as a superposition of symmetric and antisymmetric contributions. Giurgiutiu [3] found the value of $\alpha$ for a single-sided PWAS excitation to be $\alpha=4$.

Refinements of Crawley and deLuis [1] and Crawley and Anderson [2] approach have been reported in Luo and Tong [4], Tong and Luo [5], and Ryu and Wang [5]. Luo and Tong [4] and Tong and Luo [5] studied both static and dynamic solution of a piezoelectric smart beam and introduced the peel stress effect but still within the limitations of the EulerBernoulli theory of bending. Ryu and Wang [5] analyzed the interfacial stress induced by a surface-bonded piezoelectric actuator on a curved beam.

The present paper will present the extension of the work of Crawley and deLuis [1] for the case of two modes at high frequency. It will introduce the problem of deriving the interfacial shear stress between PWAS and structure when two or more wave modes are present. Tuning curves of the PWAS-structure system will be computed with both a low frequency approximation and an extended solution.

\footnotetext{
${ }^{1}$ Tel: (803) 7770619 Fax: (803) 7770106 bottai@engr.sc.edu
}

Sensors and Smart Structures Technologies for Civil, Mechanical, and Aerospace Systems 2010, edited by Masayoshi Tomizuka, Chung-Bang Yun, Victor Giurgiutiu, Jerome P. Lynch, Proc. of SPIE Vol. 7647, 76472Q - (c) 2010 SPIE · CCC code: 0277-786X/10/\$18 - doi: 10.1117/12.847676 


\section{Limits of the low frequency approximation}

The classic solution derived by Crawley and de Luis [1] was developed by taking into consideration the axial mode and the flexural mode. At low frequency, the first symmetric Lamb wave mode (S0) approximates the axial mode and its stress distribution is the same as the one assumed (see Figure 1a). Likewise, at low frequency, the first antisymmetric Lamb wave mode (A0) approximates the flexural mode and its stress distribution is the same as the one assumed (see Figure 1a). As the frequency increases, the stress distribution of the two modes is no longer linear across the thickness of the plate as shown in Figure 1b. At frequencies above the first cut-off frequency, the number of modes present in the structure is greater than two. Figure 1c shows the stress distribution of the first three Lamb wave modes for a frequencythickness product value below the first symmetric cut-off frequency, three modes are present and their stress distribution is nonlinear across the thickness. Hence, the approximation of the first two Lamb wave modes with the axial and flexural waves no longer holds. A new shear lag derivation is needed in order to verify the magnitude of the error when the classic solution is used at high frequencies.

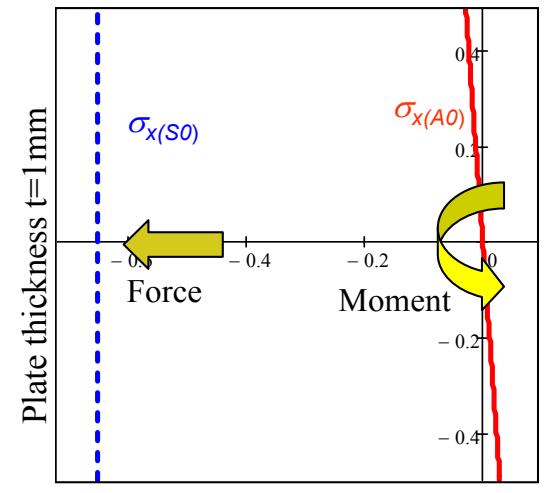

a)

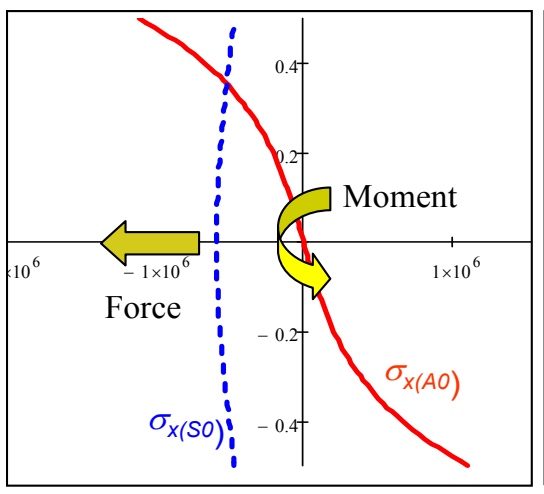

b)

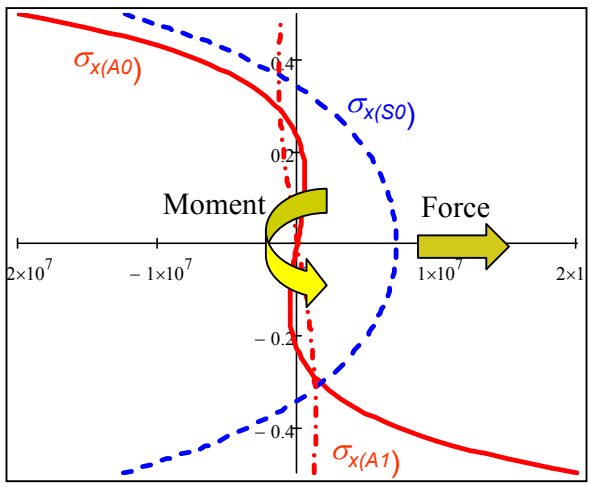

c)

Figure 1. a) Stress distributions of the first antisymmetric mode (S0) and first antisymmetric mode (A0) for low frequencies; b) Stress distribution of the first symmetric and antisymmetric modes at frequency-thickness product of $780 \mathrm{kHz} \mathrm{mm}$. c) Stress distribution of the first three Lamb wave modes (A0, S0, and A1) at frequency-thickness product of $1600 \mathrm{kHz} \mathrm{mm}$.

\section{Shear lag derivation with low frequency approximation}

Assume that two modes have been excited in a plate of thickness $2 d$ : axial mode and flexural mode. Assume also that the flexural mode stress distribution follows the Bernoulli-Euler assumption, i.e., plane sections remain plane and perpendicular to the mid-plane. As shown in Figure 1a, the axial stress has constant amplitude $\sigma_{a x}$ across the thickness and the flexural amplitude $\sigma_{\text {flex }}$ has a linear distribution across the thickness equal to $\sigma_{\text {flex }} y / d$; the total stress is given by superposition, i.e., $\sigma(x, y)=\sigma_{a x}(x)+(y / d) \sigma_{f l e x}(x)$. The stress resultants due to the stress distribution in the structure are a force and a moment, i.e.,

$$
\left\{\begin{array}{l}
N_{x}(x)=\int_{-d}^{+d} \sigma(x, y) d y=2 d \sigma_{a x}(x) \\
M_{z}(x)=\int_{-d}^{+d} \sigma(x, y) y d y=\left(2 d^{2} / 3\right) \sigma_{f l e x}(x)
\end{array}\right.
$$

Figure 2 shows the forces and the moments applied to an infinitesimal element of structure due to the presence of a PWAS bonded on the top surface and guided waves propagating in the structure. In Figure $2 \tau d x$ is the force applied from the PWAS to the structure through the bond layer. Equilibrium of the infinitesimal element of Figure 2 yields 


$$
\left\{\begin{array}{l}
N_{x}^{\prime}+\tau=0 \\
M_{z}^{\prime}+\tau d=0
\end{array}\right.
$$

where $(\cdot)^{\prime}=\partial(\cdot) / \partial x$.

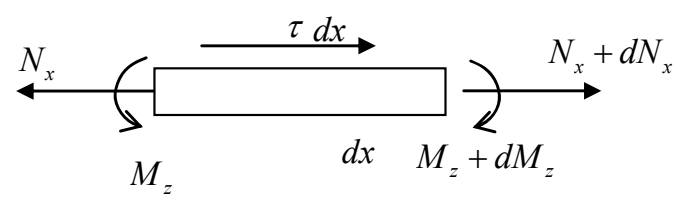

Figure 2. Equilibrium of an infinitesimal element.

Substitution of Equations (1) into Equation (2) yields

$$
\left\{\begin{array}{l}
t \sigma_{a x}^{\prime}+\tau=0 \\
t \sigma_{\text {flex }}^{\prime}+3 \tau=0
\end{array}\right.
$$

Sum up Equations (3) to get the equilibrium of the infinitesimal element, i.e. $t \sigma^{\prime}+\alpha \tau=0$, where $\sigma$ is the total stress in the structure at the upper surface, i.e., $\left.\sigma(y)\right|_{y=d}=\sigma_{a x}+\sigma_{f l e x}$, and the modal repartition number $\alpha$ is

$$
\alpha=1+3=4
$$

Note that in the modal repartition number $\alpha$, the contribution due to the symmetric mode is equal to 1 and the contribution due to the antisymmetric mode is equal to 3 . Consider the stress-strain relations in the structure and in the transducer of thickness $t_{a}$, i.e.,

$$
\left\{\begin{array}{l}
\sigma=E \varepsilon \\
\sigma_{a}=E_{a}\left(\varepsilon_{a}-\varepsilon_{I S A}\right)
\end{array}\right.
$$

where subscript $a$ denotes the stress and strain in the actuator and $\varepsilon_{I S A}$ is the induced strain by the PWAS. Using the equilibrium of the PWAS, i.e. $t_{a} \sigma_{a}^{\prime}-\tau=0$, and the equilibrium of the infinitesimal element derived before, the stressstrain relation Equation (5) yields

$$
\left\{\begin{array}{l}
t E \varepsilon^{\prime}+\alpha \tau=0 \\
t_{a} E_{a} \varepsilon_{a}^{\prime}-\tau=0
\end{array}\right.
$$

where $E$ is the Young modulus of the structure and $E_{a}$ is the Young modulus of the PWAS. Consider a bond layer between the transducer and the structure of thickness $t_{b}$ and shear modulus $G_{b}$. Through the use of the shear stress-strain relation in the bonding layer, i.e. $\tau=G_{b} \gamma=\left(G_{b} / t_{b}\right)\left(u_{a}-u\right)$ and the solution of system in Equation (6), we can derive the ordinary differential equation (ODE) of the shear stress transferred from the PWAS to the structure, i.e.,

$$
\tau^{\prime \prime}(x)-\Gamma^{2} \tau(x)=0
$$

where the shear lag parameter $\Gamma$ is defined as $\Gamma^{2}=\frac{G_{b}}{t_{b} t_{a} E_{a}} \frac{\alpha+\psi}{\psi}$, and $\psi$ is the stiffness ratio between structure and transducer, i.e. $\psi=E t / E_{a} t_{a}$. The ODE in Equation (7) can be solved applying the boundary conditions that the stress at the PWAS tips are equal to zero and the stress in the structure at the same locations are zero. Hence, solution of Equation (7) is given by 


$$
\tau(x)=\frac{\psi}{\alpha+\psi} \frac{E_{a} t_{a} \varepsilon_{I S A}}{a} \frac{\Gamma a}{\cosh \Gamma a} \sinh \Gamma x
$$

Equation (8) is the solution derived by Crawley and de Luis [1].

\section{Shear lag derivation for first two Lamb modes}

We want to derive the modal repartition number in the case of only the first symmetric and first antisymmetric modes present and for any frequency below the A1 cut-off frequency. To calculate the modal repartition number $\alpha$, we assume that there are two stress modes present, one symmetric, $\sigma_{S}(y)$, and another antisymmetric, $\sigma_{A}(y)$. At this stage we remove the assumption that the stress distributions are linear, but we retain the Bernoulli-Euler assumption. The total stress is given by superposition of the symmetric and antisymmetric modes, i.e., $\sigma(x, y)=a_{S}(x) \sigma_{S}(y)+a_{A}(x) \sigma_{A}(y)$. The stress resultants for this case are given by

$$
\left\{\begin{array}{l}
N_{x}(x)=\int_{-d}^{+d} \sigma(x, y) d y=2 d \Lambda_{S} a_{S}(x) \\
M_{z}(x)=\int_{-d}^{+d} \sigma(x, y) y d y=2 d^{2} \Lambda_{A} a_{A}(x)
\end{array}\right.
$$

where: $\Lambda_{S}$ is the symmetric modeshape constant given by $\Lambda_{S}=(1 / 2 d) \int_{-d}^{+d} \sigma_{S}(y) d y$ and it represents the average stress distribution; $\Lambda_{A}$ is the antisymmetric modeshape constant given by $\Lambda_{A}=\left(1 / 2 d^{2}\right) \int_{-d}^{+d} \sigma_{A}(y) y d y$ and it represents the equivalent stress distribution that gives the same resultant moment. Following the procedure used in Section 3 the modal repartition number assumes a new expression which depends on frequency-thickness product $f d$, i.e.,

$$
\alpha=\sigma_{S}(d) / \Lambda_{S}+\sigma_{A}(d) / \Lambda_{A}
$$

Equation (10) gives a generic expression for $\alpha$ that depends only on the stress distributions $\sigma_{S}(y)$ and $\sigma_{A}(y)$. The value of $\alpha$ determined through Equation (10) can be used to derive the shear lag parameter $\Gamma$ that is formally equal to the one derived for low frequency approximation. It is to note that, for low frequency approximation, the modal repartition number in Equation (10) reduces to 4 as in Equation (4) (for more details see Giurgiutiu and Santoni-Bottai [7]).

\section{Shear lag derivation in the presence of $N$ modes}

To derive the shear lag parameter when $N$ modes are present, we assume that the total stress is made of the superposition of the $N$ modes stress distributions, i.e., $\sigma(x, y)=\sum_{n=1}^{N} a_{n}(x) \sigma_{n}(y)$. With this assumption, the stress resultants now become

$$
\left\{\begin{array}{l}
N_{x}(x)=\int_{-d}^{+d} \sigma(x, y) d y=2 d \sum \Lambda_{n}^{S} a_{n}(x) \\
M_{z}(x)=\int_{-d}^{+d} \sigma(x, y) y d y=2 d^{2} \sum \Lambda_{n}^{A} a_{n}(x)
\end{array}\right.
$$

where the modeshape constants are now given by $\Lambda_{n}^{S}=(1 / 2 d) \int_{-d}^{+d} \sigma_{n}(y) d y$ and $\Lambda_{n}^{A}=\left(1 / 2 d^{2}\right) \int_{-d}^{+d} \sigma_{n}(y) y d y$. From the equilibrium equation of the infinitesimal element, Equation (2), we derive, as done for the low frequency approximation, the following system

$$
\left\{\begin{array}{l}
t \sum \Lambda_{n}^{S} a_{n}^{\prime}(x)+\tau(x)=0 \\
t \sum \Lambda_{n}^{A} a_{n}^{\prime}(x)+\tau(x)=0
\end{array}\right.
$$


This time the system in Equations (12) has $N$ unknowns, i.e., it is $N-2$ indeterminate. Hence, it is not possible to solve the system for $a_{n}^{\prime}(x)$. The approach used so far to derive the shear lag solution is no longer valid.

To obtain the shear lag for the case of $N$ generic modes we will express the stresses as expansions in Lamb wave modes (Auld [8]) where the modal participation factors $a_{n}(x)$ expressions are known. The total stress in the structure can be written as $\sigma(x, y)=\sum_{n=1}^{N} a_{n}^{+}(x) \sigma_{n}(y)+\sum_{n=1}^{N} a_{n}^{-}(x) \sigma_{n}(y)$ where $n$ represents the $n^{\text {th }}$ mode excited in the plate; the amplitude $a_{n}^{ \pm}$is given by

$$
a_{n}^{ \pm}(x)=\left(\tilde{v}_{x}^{n}(d) e^{\mp i \xi_{n} x} / 4 P_{n n}\right) \int_{\mp a}^{x} e^{ \pm i \xi_{n} \bar{x}} \tau(\bar{x}) d \bar{x}
$$

the plus and minus represent the forward and backward waves, respectively; $\tilde{v}_{x}^{n}(d)$ is the conjugate of the wave velocity; $\xi_{n}$ is the wave number; $P_{n n}$ is the power flow in the $x$ direction due to the $n^{\text {th }}$ wave mode defined as

$$
P_{n n}=-(1 / 2) \int_{-d}^{d}\left(\tilde{v}_{y}^{n}(y) \sigma_{x y}^{n}(y)+\tilde{v}_{x}^{n}(y) \sigma_{x x}^{n}(y)\right) d y
$$

Following Giurgiutiu and Santoni-Bottai [7], we find the modal repartition number to be

$$
\alpha=-\sum_{n=1}^{N}\left(t \tilde{v}_{x}^{n}(d) \sigma_{n}(d) / 2 P_{n n}\right)
$$

and the shear stress differential equation is now given by the integro-differential equation

$$
\tau^{\prime \prime}(x)-\Gamma^{2} \tau(x)-i \sum_{n=1}^{N} \eta_{n}\left[e^{-i \xi_{n} x} \int_{-a}^{x} e^{i \xi_{n} \bar{x}} \tau(\bar{x}) d \bar{x}+e^{i \xi_{n} x} \int_{x}^{a} e^{-i \xi_{n} \bar{x}} \tau(\bar{x}) d \bar{x}\right]=0
$$

where $\eta_{n}=\frac{G_{b}}{t_{b} E} \frac{\xi_{n} \tilde{v}_{x}^{n}(d) \sigma_{n}(d)}{4 P_{n n}}$, the shear lag is formally equal to that derived for low frequency approximation, and the modal repartition number is given by

$$
\alpha=-\frac{t_{a} E_{a}}{E} \sum_{n=1}^{N} \frac{\tilde{v}_{x}^{n}(d)}{2 P_{n n}} \sigma_{n}(d)
$$

It is to note that for low frequency approximation and Euler Bernoulli assumption, the modal repartition number in Equation (17) reduces to 4 as in Equation (4) (Giurgiutiu and Santoni-Bottai [7]).

To solve Equation (16), we apply the variational iteration method (VIM) as shown in He [9] and Wang and He [10]. Solution of the integro-differential equation yields (Giurgiutiu and Santoni-Bottai [7])

$$
\tau_{1}(x)=A \sinh \Gamma x-\sum_{n=1}^{N} \frac{\eta_{n} \Gamma \xi_{n}}{\Gamma^{2}+\xi_{n}^{2}}\left\{\begin{array}{l}
{\left[\frac{2 e^{-i \xi_{n} a}}{\Gamma^{2}+\xi_{n}^{2}}\left(\Gamma \cosh \Gamma a+i \xi_{n} \sinh \Gamma a\right)+1\right] \sinh \Gamma x} \\
-\frac{2 e^{-i \xi_{n} a}}{\xi_{n}\left(\Gamma^{2}+\xi_{n}^{2}\right)}\left(\Gamma \cosh \Gamma a+i \xi_{n} \sinh \Gamma a\right) \Gamma \sin \xi_{n} x-x \cosh \Gamma x
\end{array}\right\}
$$

where $A=G_{b} \varepsilon_{I S A} /\left(t_{b} Q\right)$ and $Q=\Gamma \cosh \Gamma a+f\left(\eta_{n}, a\right)$

\section{Modal repartition number}

In the previous sections we have derived the modal repartition number through three different methods: classic or $\alpha$ at low frequency approximation; $\alpha$ for frequencies below first cut-off frequency (A0 and S0 only); and $\alpha$ for any frequency 
( $N$ modes present). Each derivation gave a new formulation for the repartition number. In this section, we will present how the modal repartition number behaves as the frequency increases.

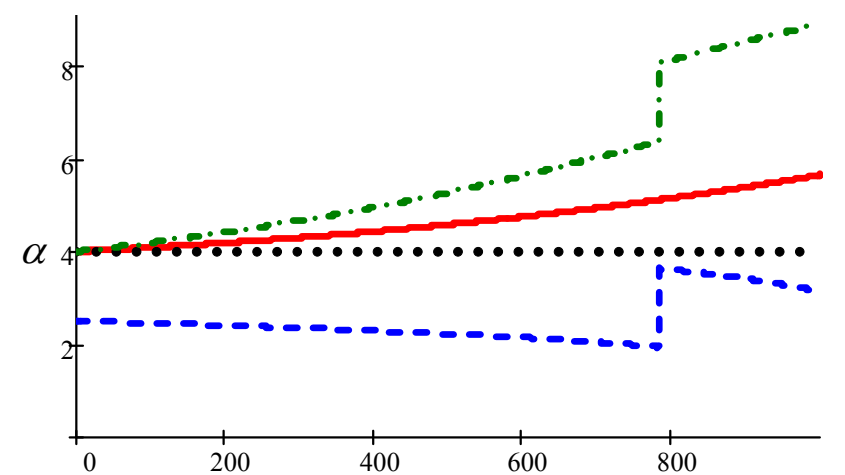

a) fd $(\mathrm{kHz} \mathrm{mm})$

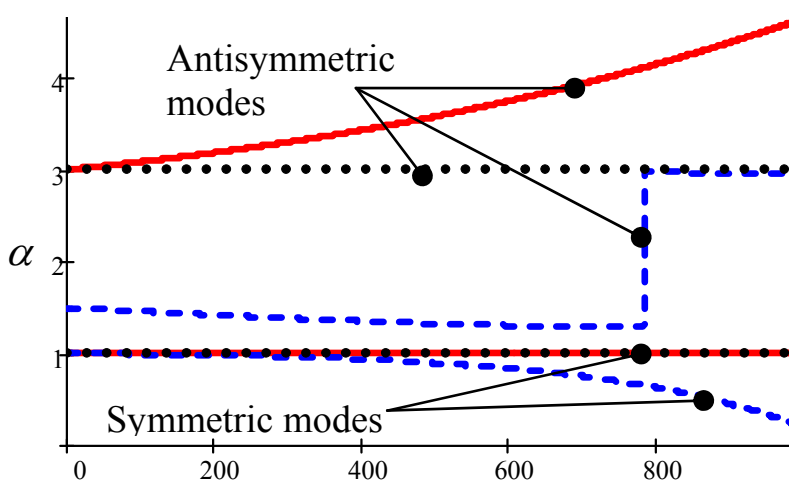

b)

Figure 3. Mode repartition number as a function of frequency. Dot line: classic solution $\alpha=4$; solid line: 2 modes solution Equation

(10) for S0 and A0; dashed line: $N$ generic mode solution Equation (17) for S0, A0, and A1; dash-dot line: $N$ generic mode solution

Equation (17) for S0, A0, and A1 and contribution form shear stress equal to zero in the power flow. a) Total repartition number. b)

Repartition number divided between $\alpha$ from the antisymmetric modes and $\alpha$ from the symmetric modes.

Figure 3a shows how the modal repartition number $\alpha$ varies with the frequency. The dotted black line is the modal repartition number derived from the classic solution; its value is a constant independent of the frequency. The solid red line represents the repartition number when only the first symmetric and antisymmetric modes are present as derived in Equation (10). The repartition number is equal to 4 at low frequencies and then it increases with the increasing frequency. It is interesting to note that while the symmetric mode contribution to the repartition number does not change with frequency (solid line in Figure 3b), the variation of $\alpha$ is entirely due to the contribution of the antisymmetric mode (solid line in Figure $3 \mathrm{~b}$ ). For frequency values beyond $\sim 800 \mathrm{kHzmm}$ this solution is no longer valid because a third mode A1 is present.

The dashed blue line in figure is the value of the repartition number derived with the $N$ generic mode formulation. From 0 to $\sim 800 \mathrm{kHz}$ only S0 and A0 are present, at the cut-off frequency, the repartition number value has a discontinuity that is due to the presence of $\mathrm{A} 1$ (only the reparation number contribution from the antisymmetric modes in Figure $3 \mathrm{~b}$ has this discontinuity). The starting value of the repartition number is 2.5 and its value decreases as the frequency increases. The presence of A1 increases the total value of the repartition number. If the product $\tilde{v}_{y}^{n}(y) \sigma_{x y}^{n}(y)$ in the antisymmetric power flow derivation (Equation (14)) is set to zero, the repartition number curve changes significantly (dashed-dot green line). The starting value is 4 as in the classic derivation and the repartition number increases with the frequency increase.

In the case of $N$ generic modes present, the contribution due to the symmetric mode is no longer constant with frequency, but decreases with it. On the other end, the contribution due to the antisymmetric mode gives an almost constant repartition number. The effect is the opposite of what as been found with the simplified derivation for 2 modes; the symmetric mode affects the variation of the total repartition number.

\section{Tuning curves}

Under electric excitation, the PWAS undergoes oscillatory contractions and expansions which are transferred to the structure through the bonding layer and thus excite Lamb waves into the structure. In this process, several factors influence the behavior of the excited wave: the thickness of the bonding layer, the geometry of the PWAS, the thickness and material of the structure. The result of the influence of all these factors is the tuning of the PWAS with various Lamb wave modes in the material. This phenomenon has been studied by Giurgiutiu [11] who developed the theory of the 
interaction of a rectangular PWAS with straight-crested Lamb waves. Lately, Raghavan and Cesnik [12] extended these results to the case of a circular transducer coupled with circular-crested Lamb waves.

Giurgiutiu [11] developed a plane-strain analysis of the PWAS-structure interaction using the space-domain Fourier analysis; in this analysis the strain and displacement wave solutions for straight crested waves excited by a rectangular PWAS were studied. Under the assumption of $z$-invariant motion, hence the PWAS is infinite in the $z$ direction, the expression developed for strain is:

$$
\left.\varepsilon_{x}(x, t)\right|_{y=d}=\frac{1}{2 \mu}\left[\sum_{\xi^{S}} \frac{\tilde{\tau} N_{S}\left(\xi^{S}\right)}{D_{S}^{\prime}\left(\xi^{S}\right)} e^{i\left(\xi^{S} x-\omega t\right)}+\sum_{\xi^{A}} \frac{\tilde{\tau} N_{A}\left(\xi^{A}\right)}{D_{A}^{\prime}\left(\xi^{A}\right)} e^{i\left(\xi^{A} x-\omega t\right)}\right]
$$

where $N_{S}=\xi \beta\left(\xi^{2}+\beta^{2}\right) \cos (\alpha d) \cos (\beta d) ; \quad N_{A}=\xi \beta\left(\xi^{2}+\beta^{2}\right) \sin (\alpha d) \sin (\beta d), D_{S}$ and $D_{A}$ are the Rayleigh-Lamb equation for symmetric and antisymmetric mode respectively, $\xi^{S}$ and $\xi^{4}$ are the zeros of $D_{S}$ and $D_{A}$ respectively, and $\tilde{\tau}$ is the Fourier transform of the external excitation.

For the low frequency approximation, the excitation provided by a PWAS bonded on the top surface of a plate through an adhesive layer is represented by Equation (8). If we take in consideration the case of an ideal bond, we assume that the bond layer thickness is as thin as possible, i.e. $t_{b} \rightarrow 0$. As $t_{b} \rightarrow 0$ the shear lag parameter goes to infinity; at the limit case, the shear stress transmitted by the PWAS can be represented through the pin-force model, i.e.,

$$
\tau(x, d)= \begin{cases}\tau_{0}[\delta(x-a)-\delta(x+a)] & \text { if }|x| \leq a \\ 0 & \text { if }|x|>a\end{cases}
$$

where $\tau_{0}=[\psi /(\alpha+\psi)]\left(E_{a} t_{a} / a\right) \varepsilon_{I S A}$. The Fourier transform of Equation (20) is a function that depends on the PWAS size, $2 a$, and the modal wavenumber, $\xi_{n}$, i.e.,

$$
\tilde{\tau}=\int_{-a}^{a} \tau(x, d) e^{i \xi_{n} x} d x=2 i \tau_{0} \sin \xi_{n} a
$$

If we remove the assumption of ideal bonding, the excitation provided by the PWAS is given by Equation (8), i.e., the shear lag model of stress transfer between the PWAS and the structure. The excitation can be written as

$$
\tau(x, d)= \begin{cases}\tau_{1} \sinh \Gamma x & \text { if }|x| \leq a \\ 0 & \text { if }|x|>a\end{cases}
$$

where $\tau_{1}=\tau_{0} \Gamma a / \cosh \Gamma a$. The Fourier integral of the excitation is a function that depends on the PWAS size, $2 a$, the modal wavenumber, $\xi_{n}$, and shear lag parameter, $\Gamma$, i.e.,

$$
\tilde{\tau}_{n}\left(\xi_{n}\right)=\int_{-a}^{a} \tau(x, d) e^{i \xi_{n} x} d x=2 i \frac{\Gamma \sin \xi_{n} a \cosh \Gamma a-\xi_{n} \sinh \Gamma a \cos \xi_{n} a}{\Gamma^{2}+\xi_{n}^{2}}
$$

Note that the ideal bonding solution is the limit case of the shear lag solution as $\Gamma$ goes to infinity.

For the case of $N$ modes excited in the structure, consider the shear stress in the bonding layer as derived in Equation (18). The Fourier integral of the excitation is a function that depends on the relation between the PWAS size, $2 a$, the modal wavenumber, $\xi_{n}$, and shear lag parameter $\Gamma$, and the number of modes present in the structure, i.e., 


$$
\begin{array}{r}
\tilde{\tau}\left(\xi_{n}\right)=\frac{2 i}{\Gamma^{2}+\xi_{n}{ }^{2}}\left\{\Gamma^{2}-\sum_{m=1}^{M} \frac{\eta_{m} \xi_{m}}{\Gamma^{2}+\xi_{m}{ }^{2}}\left[\frac{2 \Gamma e^{-i \xi_{m} a}}{\Gamma^{2}+\xi_{m}{ }^{2}}\left(\begin{array}{l}
\Gamma \cosh \Gamma a \\
+i \xi_{m} \sinh \Gamma a
\end{array}\right)+1\right]\right\}\left(\begin{array}{l}
\Gamma \sin \xi_{n} a \cosh \Gamma a \\
-\xi_{n} \cos \xi_{n} a \sinh \Gamma a
\end{array}\right) \\
+\sum_{m=1}^{M} \frac{\eta_{m} \Gamma}{\Gamma^{2}+\xi_{m}{ }^{2}}\left[\begin{array}{l}
\frac{2 i e^{-i \xi_{m} a}}{\Gamma^{2}+\xi_{m}{ }^{2}}\left(\Gamma \cosh \Gamma a+i \xi_{m} \sinh \Gamma a\right) \Gamma\left(\frac{\sin \left(\xi_{m}-\xi_{n}\right) a}{\left(\xi_{m}-\xi_{n}\right)}-\frac{\sin \left(\xi_{m}+\xi_{n}\right) a}{\left(\xi_{m}+\xi_{n}\right)}\right) \\
+\frac{2 i \xi_{m} a}{\Gamma^{2}+\xi_{n}{ }^{2}}\left(\Gamma \sin \xi_{n} a \sinh \Gamma a-\xi_{n} \cos \xi_{n} a \cosh \Gamma a\right) \\
-\frac{\xi_{m}}{2}\left(\frac{e^{\left(\Gamma+i \xi_{n}\right) a}-e^{-\left(\Gamma+i \xi_{n}\right) a}}{\left(\Gamma+i \xi_{n}\right)^{2}}+\frac{e^{-\left(\Gamma-i \xi_{n}\right) a}-e^{\left(\Gamma-i \xi_{n}\right) a}}{\left(\Gamma-i \xi_{n}\right)^{2}}\right)
\end{array}\right]
\end{array}
$$

\section{Theoretical and experimental results}

Pitch-catch experiments were performed in which one PWAS served as a transmitter and another PWAS served as a receiver. The predicted values were compared with the experimental results. The signal used in the experiments was a Hanning-windowed tone burst with 3 counts. The signal was generated with a function generator (Hewlett Packard 33120A) and sent through an amplifier (Krohn-Hite model 7602) to the transmitter PWAS. A data acquisition instrument (Tektronix TDS5034B) was used to measure the signal measured by the receiver PWAS. The PWAS were permanently bonded to a plate made of aluminum alloy 2024-T3 with thickness $1 \mathrm{~mm}$ and size 1200x1060 mm. The distance between the PWAS was $250 \mathrm{~mm}$. The frequency of the signal was swept from 10 to $700 \mathrm{kHz}$ with steps of $20 \mathrm{kHz}$. At each frequency, we collected the wave amplitude and the time of flight for both the symmetric mode and the antisymmetric modes.

Figure 4a shows the experimental and theoretical tuning curves for the first antisymmetric and symmetric modes. The amplitude of the theoretical curves have been scaled such as the first antisymmetric peak amplitude was the same as the experimental one (multiplication factor is 5.3 for Equation (21) and 2.6 for both Equation (23) and Equation (24)).
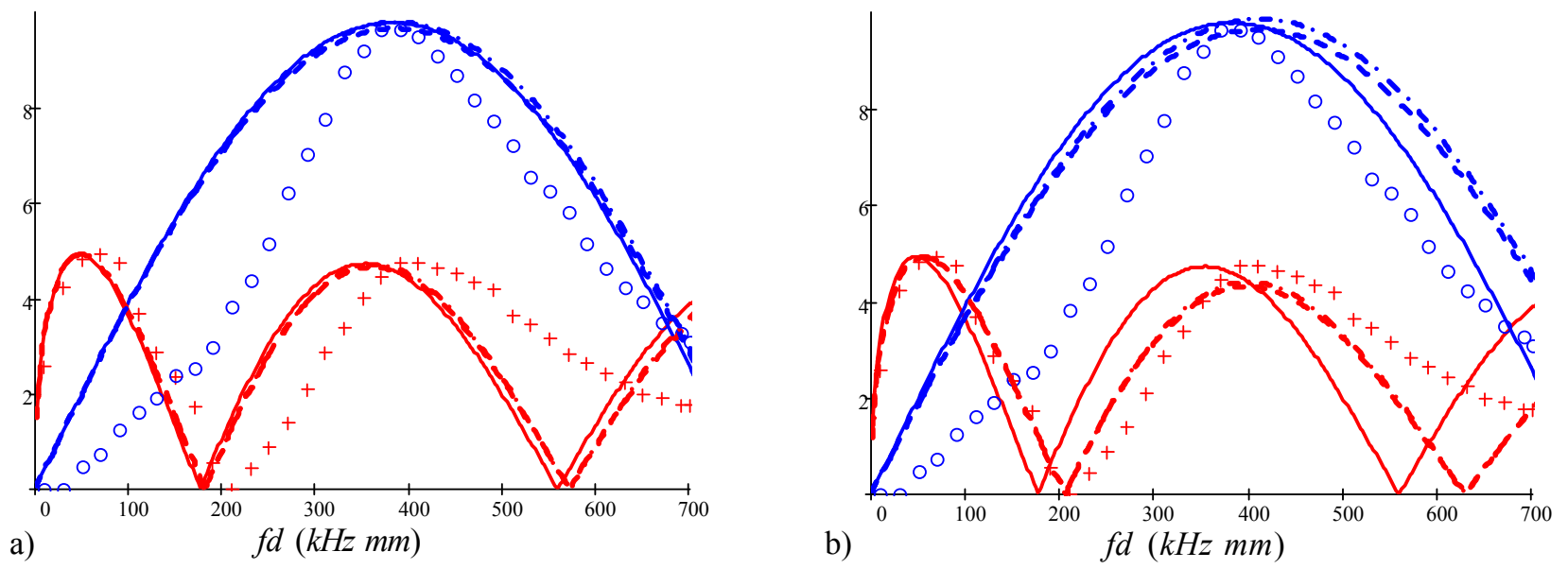

Figure 4. Tuning curves for an Aluminum plate $1 \mathrm{~mm}$ thick and a $7 \mathrm{~mm}$ square PWAS. Blue circles: Experimental S0 mode data; Red crosses: Experimental A0 mode data; Solid line: theoretical A0 (red) and S0 (blue) values from Equation (21); Dash line: theoretical A0 (red) and S0 (blue) values from Equation (23); Dash dot line: theoretical A0 (red) and S0 (blue) values from Equation (24). a)

Bond thickness $1 \mu \mathrm{m}$; b) bond thickness $30 \mu \mathrm{m}$.

In Figure 4a both the maxima and the zeros of the antisymmetric theoretical curves are not in the same locations of the experimental ones, while the symmetric prediction curves are more close to the expected values. The prediction curves 
derived with Equations (23) and (24) are almost coincident for any frequency and they are closer to the solution through ideal bonding assumption at the low frequencies.

In Figure $4 \mathrm{~b}$, the predicted curves are plotted for a thicker bond thickness $\left(t_{b}=30 \mu \mathrm{m}\right)$. The first antisymmetric maxima and minimum are now coincident with the experimental values, while the symmetric maxima have not changed their location significantly. As in Figure 4a, there is almost no difference between the predictions made through Equation (23) or Equation (24) for thicker bonds.

In Santoni-Bottai and Giurgiutiu [13] it was noticed that the best concordance between the experimental data and the predicted curves through Equation (21) was achieved for theoretical PWAS length smaller than that of the real transducer. In particular it was found that the smaller the PWAS, the greater was the percentage of non effective transducer. In Table 8.1 are reported the values of the real PWAS lengths, the theoretical values used, the percentages of the effective length of the real PWAS, and the complimentary non effective length. While the rectangular PWAS along its greatest dimension $(25 \mathrm{~mm})$ transmits the stress to the plate very close to its borders, the same PWAS in the smallest dimension $(5 \mathrm{~mm})$ transmits the stress to the plate at $5 \%$ of its length before the borders. The adjustment of the real PWAS length was necessary because in the development of the theory, it was supposed that the stress induced by the PWAS was transferred to the structure at the end of the PWAS itself.

Table 8.1: Actual and effective PWAS length (Santoni-Bottai and Giurgiutiu [13])

\begin{tabular}{|c|c|c|c|}
\hline Real PWAS length & Effective PWAS length & \% of effective PWAS & \% of non effective PWAS \\
\hline $25 \mathrm{~mm}$ & $24.8 \mathrm{~mm}$ & $99.2 \%$ & $0.8 \%$ \\
\hline $7 \mathrm{~mm}$ & $6.4 \mathrm{~mm}$ & $91.4 \%$ & $8.6 \%$ \\
\hline $5 \mathrm{~mm}$ & $4.5 \mathrm{~mm}$ & $90 \%$ & $10 \%$ \\
\hline
\end{tabular}

By changing the PWAS effective length from $7 \mathrm{~mm}$ to $6.4 \mathrm{~mm}$ in Equation (21), the minima of the solid curve in Figure $4 \mathrm{~b}$ would be in the same location as for those of the experimental values.

\section{Conclusion}

It has been shown that as the frequency-thickness product increases, the classic shear lag solution with modal repartition number $\alpha=4$ is no longer valid. An extension to the higher frequency has been presented and a first iterative solution has been derived. The exact solution for $\mathrm{N}$-generic modes gives the same result as the classical shear-lag solution under the Euler-Bernoulli assumption. However, the exact solution shows that the contribution due to the shear in the antisymmetric mode leads to a different value of the modal repartition number. The iterative solution derived for $N$ generic modes can be used to derive the interfacial shear stress between PWAS and structure at any frequency and in the presence of two or more Lamb wave modes. The major effect of the higher frequencies is to increase the area of the PWAS that contributes to the transmission of the shear. This effect on the shear transmission affects the use of ideal bonding assumption in the derivation of the tuning between PWAS and structure and requires a correction factor as shown in Santoni-Bottai and Giurgiutiu [13]. Hence, the prediction curve derived through Equation (23) or (24) could be used to verify the thickness of the bonding between the PWAS and structure.

\section{Acknowledgment}

Financial support from National Science Foundation awards \#CMS 0408578 and \#CMMI 0925466, Dr. Chi Shih Liu Program Director, is thankfully acknowledged.

\section{References}

1. Crawley, E. F.; De Luis, J. (1987) "Use of Piezoelectric Actuators as Elements of Intelligent Structures", AIAA Journal, Vol. 25, No. 10, pp. 1373-1385, 1987

2. Crawley, E. F.; Anderson, E. H. (1990) "Detailed Models of Piezoceramic Actuation of Beams", Journal of Intelligent Material Systems and Structures, Vol. 1, No. 1, Jan. 1990, pp. 4-25 
3. Giurgiutiu, V. (2005) "Tuned Lamb Wave Excitation and Detection with Piezoelectric Wafer Active Sensors for Structural Health Monitoring", Journal of Intelligent Material Systems and Structures, Vol. 16, No. 4, Apr. 2005, pp. 291-305

4. Luo, Q.; Tong, L. (2002), "Exact static solutions to piezoelectric smart beams including peel stresses", International Journal of Solids and Structures, Vol. 39, 2002, pp. 4677-4722

5. Tong, L.; Luo, Q. (2003) "Exact dynamic solutions to piezoelectric smart beams including peel stresses", International Journal of Solids and Structures, Vol. 40, 2003, pp. 4789-4836

6. Ryu, D. H.; Wang K. W. (2004) "Analysis of interfacial stress and actuation authorities induced by surface-bonded piezoelectric actuators on curved flexible beams", Smart Materials and Structures, Vol. 13, No. 4, August 2004, pp. $753-761$

7. Giurgiutiu, V.; Bottai-Santoni, G. (2009) "An Extension of the Shear Lag Solution for Structurally Attached Ultrasonic Active Sensors”, AIAA Journal, vol. 47, issue 8, pp. 1980-1983, Technical Notes, August 2009

8. Auld, B. A. (1990) “Acoustic Fields and waves in solids", John Wiley \& Son Vol. 1 and 2, 1990

9. He, J.H., (2007) "Variational iteration method-Some recent results and new interpretations", Journal of Computational and Applied Mathematics, 207, pp 3-17

10. Wang, S. Q; He, J. H., (2007) "Variational iteration method for solving integro-differential equations" Physics Letters A 367, pp 188-191

11. Giurgiutiu, V. (2003) "Lamb Wave Generation with Piezoelectric Wafer Active Sensors for Structural Health Monitoring", SPIE'S $10^{\text {th }}$ Annual International Symposium on Smart Structures and Materials and $8^{\text {th }}$ Annual International Symposium on NDE for Health Monitoring and Diagnostics, 2-6 March 2003, San Diego, CA, paper \# 5056-17

12. Raghavan A., Cesnik C. E. S.; "Modeling of piezoelectric-based Lamb-wave generation and sensing for structural health monitoring"; Proceedings of SPIE - Volume 5391 Smart Structures and Materials 2004: Sensors and Smart Structures Technologies for Civil, Mechanical, and Aerospace Systems, Shih-Chi Liu, Editor, July 2004, pp. 419430

13. Santoni-Bottai, G.; Giurgiutiu, V., "Simulation of the Lamb Wave Interaction between Piezoelectric Wafer Active Sensors and Host Structure", SPIE's 12th International Symposium on Smart Structures and Materials and 10th International Symposium on NDE for Health Monitoring and Diagnostics, Sensors and Smart Structures Technologies for Civil, Mechanical, and Aerospace Systems Conference, San Diego, CA, San Diego, CA, paper \# 5765-29, 6-10 Mar. 2005 